11th World Congress on Computational Mechanics (WCCM XI)

5 th European Conference on Computational Mechanics (ECCM V)

6th European Conference on Computational Fluid Dynamics (ECFD VI)

July 20-25, 2014, Barcelona, Spain

\title{
ENERGY-CONSISTENT TIME INTEGRATION FOR NONLINEAR VISCOELASTICITY
}

\author{
Sergio Conde Martín ${ }^{1}$, Juan Carlos García Orden², Ignacio Romero ${ }^{3}$ \\ ETSI Caminos, Technical University of Madrid, c/Profesor Aranguren s/n, 28040 Madrid, \\ Spain, sergio.conde@upm.es \\ ETSI Caminos, Technical University of Madrid, c/Profesor Aranguren s/n, 28040 Madrid, \\ Spain, juancarlos.garcia@upm.es \\ ETSI Industriales, Technical University of Madrid, c/José Gutierrez Abascal 2, 28006 Madrid, \\ Spain, ignacio.romero@upm.es
}

Key words: Time integration, structure preservation, geometric integration, thermodynamics, viscoelasticity

This work is concerned with the numerical solution of the evolution equations of thermomechanical systems, in such a way that the scheme itself satisfies the laws of thermodynamics. Preserving structure integrators are widely developed for conservative (Hamiltonian) systems, being the most representative method the well-known energy-momentum due to Simó and Tarnow [1]. Recently, after the works of Romero I. [2], these ideas are being used in evolution system with irreversible processes, i.e non-conservative, such as those with internal dissipation mechanisms (viscoelasticity, plasticity, damage). In this context, the structure meant to be preserved is understood in the thermodynamical way, namely, the integrator must intrinsically satisfy the laws of thermodynamics as in conservative problems this one preserved the Hamiltonian.

Within this framework, we present a novel integration method for the dynamics of an isothermal viscoelastic continuum body. This method exactly preserves the continuum laws of thermodynamics intrinsically, plus equations symmetries. The resulting solutions are physically accurate since they preserve fundamental physical properties of the model. What is more, they show an excellent performance with respect to the methods robustness and stability. Proof for these claims will be provided in the presentation as well as numerical examples that illustrate the method's performance.

The viscoelastic behaviour is modeled according to Holzapfel and Simó [3] (regardless of temperature). Such model is based on large-strain deformations. Our approach is based on a double semidiscretisation, spatial (FE-based) and temporal, being on the latter one we do the main contribution. 


\section{REFERENCES}

[1] J.C. Simó, N. Tarnow. The discrete energy-momentum method. Conserving algorithm for nonlinear elastodynamics. Zeitschriftfür Angewandte Mathematik und Physik (ZAMP), Vol. 43(5), 757-792, 1992.

[2] Ignacio Romero. Algorithms for coupled problems that preserve symmetries and the laws of thermodynamics Part I: Monolithic integrators and their application to finite strain thermoelasticity. Computer Methods in Applied Mechanics and Engineering, Vol. 199, 1841-1858, 2010.

[3] G.A. Holzapfel, J.C. Simó. A new viscoelastic constitutive model for continuous media at finite thermomechanical changes. International Journal of Solids and Structures, Vol. 33(20), 3019-3034, 1996. 